\title{
LES study of a turbulent spray jet: mesh sensitivity, mesh-parcels interaction and injection methodology
}

\author{
D'Ausilio Alessandro • Ivana Stankovic • \\ Bart Merci
}

5

6 Received: date / Accepted: date

\begin{abstract}
This study focuses on Large Eddy Simulations (LES) in the EulerianLagrangian framework of turbulent spray jets. The choice of the numerical grid relates both to turbulence level description and parcels-grid cells interaction. The objective of the present work is to determine a case set-up capable of predicting flow fields of a turbulent n-heptane spray jet for which a set of experimental data in non-reactive conditions (isothermal) is available [21]. The study first focuses on the LES grid, based on simulation with only gas phase, and subsequently on the choice of the suitable number of parcels to describe the spray. The droplets behavior is analyzed using the particle Stokes number at different axial locations. Furthermore, a variation in the existing injection methodology, as available in OpenFOAM ${ }^{\circledR}$, reveals a beneficial impact on the prediction of the experimental data.
\end{abstract}

Keywords Turbulence · Sprays · Jets · LES · Eulerian-Lagrangian · Parcels · Particle Stokes · OpenFOAM ${ }^{\circledR}$

\section{Introduction}

A. D'Ausilio, I. Stankovic and B. Merci

Department of Flow, Heat and Combustion Mechanics, Ghent University,

St. Pietersnieuwstraat 41, B-9000 Ghent, Belgium

E-mail: alessandro.dausilio@ugent.be 
the turbulent flow field structure. Preferential concentration occurs when droplets deviate from their trajectory in regions where the strain rate is high and the vorticity is low [27].

Experimental and numerical research aims at in-depth understanding of the aforementioned phenomena. However, for many applications, extensive measurements can be difficult and expensive. As an alternative, Computational Fluid Dynamics (CFD) codes have become important tool during the past decades, due to the increasing computional resources and due to the development of scalable CFD solvers (e.g, OpenFOAM ${ }^{\circledR} 2.4 .0$ [26] used in this study). Furthermore, the need for accurate simulation results of turbulent two-phase, dispersed flows, that can capture the complexity of the flow field within acceptable computational costs, has led to a significant interest in Large Eddy Simulations (LES) [16],[1],[14],[11]. In the present work, LES is employed in combination with the Eulerian-Lagrangian (EL) point particle approach technique: the gas is solved using the continuum assumption and the droplets are tracked on their way through the flow field. The tracking of all the spray particles is often computationally not affordable and in order to overcome this limitation, the strategy of clustering groups of droplets having the same properties is used, identifying them as material points named 'parcels' [10].

In order to account for the interaction between phases, i.e., momentum exchange and heat as well as mass transfer, the conservation equations of the continuous phase have to be extended by appropriate source/sink terms resulting from the dispersed phase. This coupling method is referred to as two-way coupling [4], [5]. Because of these sources/sink terms, the number of parcels and the mesh grid size appear to be closely linked. In numerical studies for internal combustion engines $[19],[20]$ a change in liquid spray penetration is observed when the mesh is refined and the number of parcels to inject during the whole injection duration is kept constant. This behavior has been explained as a statistical error due to lack of particles in many cells [18], [7]. It is therefore evident that the number of parcels representative of the real spray during the injection duration has to be chosen according to the mesh cell size. The latter has to be sufficiently small to be in the inertial subrange so that the energy-containing contribution is directly resolved and the dissipation-range contribution is modeled [15]. Inadequate choices could affect numerical results, influencing the analysis where more phenomena have to be considered, i.e, in turbulent spray combustion simulations. Therefore, the first two objectives of the present study are: a) performing a grid sensitivity study, and b) the assessment of the relation between the grid size and parcels number, for turbulent spray jets simulations.

When comparing simulation results to experimental data, it becomes clear that fine-tuning is often needed to inject parcels such that the real injection process is mimicked as closely as possible to the real injection process. This is shown in [12], introducing a conditional injection model to perform LES simulation of an ethanol spray flame, showing limitations of the default OpenFOAM ${ }^{\circledR}$ injection model to resemble the spray at hand. The third objective of the present study is to assess the importance of the injection methodology, particularly its potential impact on the preferential droplet concentration and on the turbulent gas flow field. To this purpose, the injection model, available in OpenFOAM ${ }^{\circledR}$, has been modified and results are compared to the original methodology.

The paper is structured as follows. The first and second section deal with the de- 
scription of the experimental set-up and flow field features of annular jets respectively. The third section deals with the properties of the dispersed phase flow under examination and the mathematical modeling for both gas and liquid phase. The fourth section is concerned with the numerical set-up and the injection methodologies. This is followed by the discussion of the numerical results: first an LES sensitivity analysis considering only the gas phase; then the flow field analysis including both gas and liquid, exploring the interaction between them; numerical results are validated through comparison with experimental data; and eventually the limitation of the default OpenFOAM ${ }^{\circledR}$ injection methodology through comparison to results obtained with the modified injection methodology is discussed.

\section{Experimental set-up}

A turbulent n-heptane spray jet, issuing from a central nozzle surrounded by an annular turbulent non-swirling jet, is considered in this work. The set-up belongs to the Coria Rouen Spray Burner (CRSB) database[21], [25], [24], [13]. This case is suitable for validation because the boundary conditions are well characterized and measurements of droplet fields and gas velocity are available.

The features of the CRSB and the spray nozzle characteristics are reported in Table 1. The subscripts $c$ and $d$ refer to carrier and disperse phase, respectively. The database includes measurements for gas and liquid droplet velocity, droplet diameter and droplet temperature, both for reactive and non-reactive conditions. The study of the jet in reactive conditions is beyond the scope of the present paper. The fuel injection system consists of a simplex atomizer which produces a hollow cone spray (most of the droplets are located at the edge of the conical spray pattern) with half spray angle of $40^{\circ}$. The complex injector geometry, reported in Figure 1, is in this study replaced by an annular cylinder.

Table 1 Coria Rouen Spray Burner Features

\begin{tabular}{ll}
\hline Inlet fuel conditions $\left(\dot{m}_{d}\right)$ & $0.28 \mathrm{~g} / \mathrm{s}(\mathrm{T}=298 \mathrm{~K})$ \\
Inlet (co-flow) air conditions $\left(\dot{m}_{c}\right)$ & $6 \mathrm{~g} / \mathrm{s}(\mathrm{T}=298 \mathrm{~K})$, non-swirling \\
Fuel & n-Heptane \\
Oxidizer & air \\
Type nozzle & Simplex Fuel Injector \\
Nozzle diameter & $200 \mu \mathrm{m}$ \\
Nominal Spray angle & $80^{\circ} \mathrm{hollow}$ cone \\
Inner co-flow diameter & $0.01 \mathrm{~m}$ \\
Outer co-flow diameter & $0.02 \mathrm{~m}$ \\
Reynolds Number $\left(\rho_{c} u_{c} d_{h} / \mu_{c}\right)$ & 13800 \\
Spray droplets size & {$[0.5-65 \mu m]$} \\
\hline
\end{tabular}




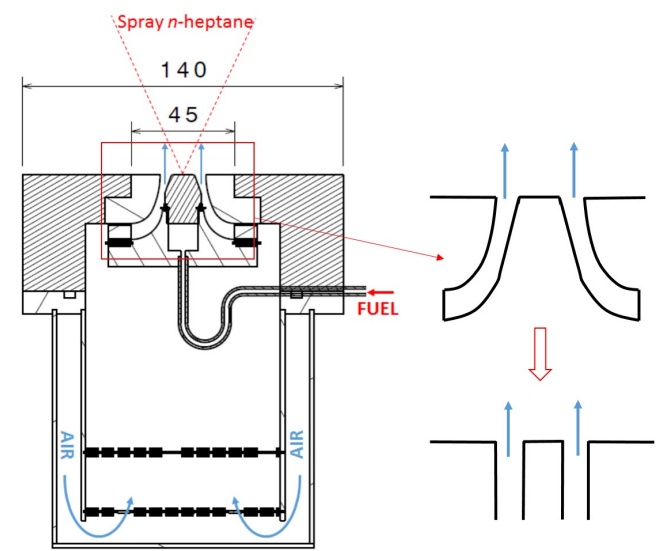

Fig. 1 Left: detail of the experimental injection system (Courtesy of [23]). Right: simplification as annular cylinder in the present work. 


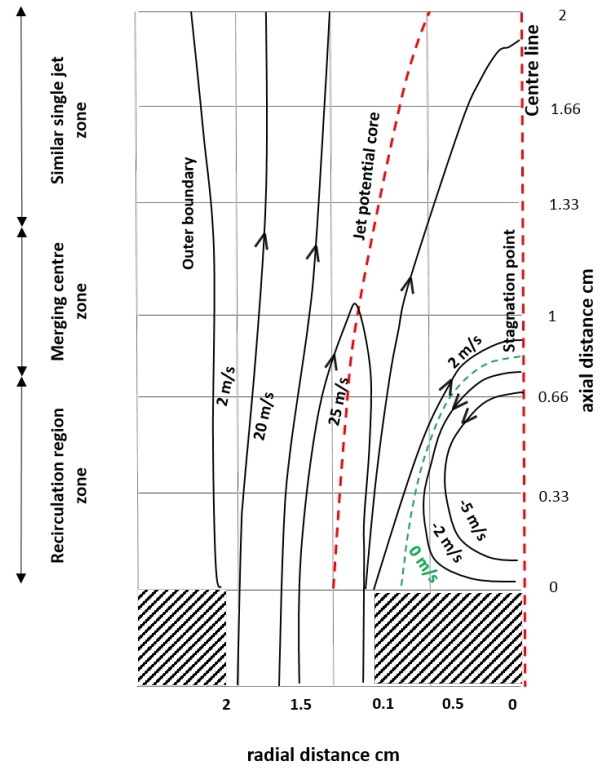

Fig. 2 Flow field features in annular jets. Sketch based on numerical results performed in this study.

The main flow features for jets issuing from an annular nozzle are sketched in Figure 2. The streamlines in the figure are based on preliminary calculations performed during this study. Chigier et al. [2] examined the flow region near the nozzle in double concentric jets. In the absence of a central jet, the annular jet issues in a quiescent environment. The shearing action between the fluid and the stagnant surrounding fluid generates a low-pressure region with negative velocities. This region is referred as "central trough" region [3]. The length of the vortex region depends on the nozzle geometry and the jet velocity.

In Figure 2, the iso-line labeled " $0 \mathrm{~m} / \mathrm{s}$ " intersects the center line at the stagnation point. The jet, initially with a double off center peaks shape, converges towards the center and beyond the stagnation point the jet velocity profiles show trends similar to that of a single jet issuing from the center.

Maximum velocities are found in the jet potential core, which depends on the shape of the nozzle geometry and the intensity of turbulence generated ahead of the exit from the nozzle. 
3 Modeling approaches

3.1 Properties of the disperse phase flow

Before introducing the governing equations, the choice of Large Eddy Simulation (LES) together with the Eulerian/Lagrangian (EL) point particle approach for disperse phase flow is discussed. According to Crowe et al. [4], a dispersed flow can be considered as dilute if the droplet diameter satisfies the following condition:

$$
d_{p}<\frac{1.33 \mu_{c}}{Z \rho_{c} \sigma}
$$

In Eq.(1), $\mu_{c}$ is the carrier phase viscosity, $\rho_{c}$ the carrier phase mass density and $\sigma$ the particle velocity fluctuation. $\mathrm{Z}$ is defined as mass flow rate ratio between dispersed and carrier phase:

$$
Z=\frac{\dot{m}_{d}}{\dot{m}_{c}}
$$

Using the data given in Table 1, it follows that for the case at hand the two-phase flow can be considered dilute if the droplet diameter is smaller than about $120 \mu \mathrm{m}$. From the experiments it is known that the droplet size range of the spray after the secondary atomization process is between 0.5 and $65 \mu \mathrm{m}$. This justifies the assumption of negligible interaction among particles due to collision. In the dilute regime, the flow of the carrier phase can be affected by the liquid phase (twoway coupling) or not (one-way coupling). We have adopted the two-way coupling approach considering that the droplet mass loading is not negligible.

It is confirmed below that the droplet injection modifies the gas flow field.

\subsection{Gas and Liquid phase formulations}

The LES equations for mass and momentum for dispersed two-phase flows read:

$$
\frac{\partial \bar{\rho}}{\partial t}+\frac{\partial \bar{\rho} \tilde{u_{j}}}{\partial x_{j}}=\bar{S}_{\rho}
$$

$$
\frac{\partial \bar{\rho} \tilde{u}_{i}}{\partial t}+\frac{\partial \bar{\rho} \tilde{u}_{i} \tilde{u}_{j}}{\partial x_{j}}=-\frac{\partial \bar{p}}{\partial x_{i}}+\frac{\partial \tau_{i j}}{\partial x_{j}}+\frac{\partial \tau_{i j}^{s g s}}{\partial x_{j}}+\bar{\rho} g_{i}+\bar{S}_{u_{i}}, i=1,2,3
$$

In Eqs.(3) and (4), $\bar{\rho}$ is the filtered density and $\tilde{u}$ is the Favre filtered velocity. The third term on the right hand side of Eq.(4) is the sub-grid scale (SGS) stress tensor that arises from the residual motions. The classical eddy-viscosity approach is followed:

$$
\tau_{i j}^{s g s}=\bar{\rho}\left(\widetilde{u_{i} u_{j}}-\tilde{u_{i}} \tilde{u_{j}}\right)=-2 \mu_{t} \tilde{S}_{i j}
$$

with the strain rate tensor, $\tilde{S}_{i j}$ :

$$
\tilde{S}_{i j}=\frac{1}{2}\left(\frac{\partial \tilde{u}_{i}}{\partial x_{j}}+\frac{\partial \tilde{u}_{j}}{\partial x_{i}}\right)
$$

The turbulent sgs viscosity, $\mu_{t}$, is modeled through the constant Smagorinsky model:

$$
\mu_{t}=\bar{\rho}\left(C_{s} \Delta^{2}\right)\left(2 \tilde{S}_{i j} \tilde{S}_{i j}\right)^{\frac{1}{2}}
$$


In Eq.(7) the filter width, $\Delta$, is the cubic root of the mesh cell volume and the constant $C_{s}$ is chosen equal to 0.167 [15]. Sensitivity studies showed no differences in the results when a dynamic Smagorinsky model is used (not shown).

The last terms on the right hand sides of Eq.(3) and Eq.(4) are source terms accounting for the coupling between liquid and gas phase. $\bar{S}_{\rho}$ is the mass source term due to evaporation [22]:

$$
\bar{S}_{\rho}=\frac{1}{V_{\text {cell }}} \sum_{k=1}^{N} n_{p, k} \dot{m}_{p, k}
$$

In Eq.(8) $V_{\text {cell }}$ is the cell volume, $N$ is the total number of parcels present in the cell during each time step, $n_{p, k}$ is the number of droplets per parcel and $\overline{\dot{m}}_{p, k}$ the mass evaporation rate for each parcel in the cell at hand. The latter is calculated as:

$$
\dot{m}_{p, k}=-2 \pi d_{p, i} \bar{\rho}_{c} D_{d} \ln \left(B_{M}+1\right)
$$

In Eq.(9), $d_{p, i}$ is the droplet diameter, $D_{d}$ is the diffusivity of the liquid into the gas phase and $B_{M}$ is calculated as follows:

$$
B_{M}=\frac{X_{S}-X_{\mathrm{inf}}}{1-X_{S}}
$$

In Eq.(10) $X_{S}$ is the liquid molar fraction at the surface and $X_{\text {inf }}$ the liquid molar fraction in the gas phase.

$\bar{S}_{u_{i}}$ represents the momentum source term due to mass evaporation and momentum exchange between phases. It is calculated as [22]:

$$
\bar{S}_{u_{i}}=\frac{1}{V_{\text {cell }}} \sum_{k=1}^{N} n_{p, k}\left(m_{p, k} \tau_{p}^{-1}\left(U_{p, i}-\tilde{U}_{\text {seen }, i}\right)+\dot{m}_{p, k} U_{p, i}\right), i=1,2,3
$$

The gas velocity at the parcel position, $\tilde{U}_{\text {seen }}$ is calculated by interpolation, using the parcel position and the cell center of the cell at hand [11]. It is assumed that $U_{\text {seen }}=\tilde{U}_{\text {seen }}$ and no dispersion model is considered.

The particle momentum equation for each parcel reads [22]:

$$
\frac{d U_{p, i}}{d t}=\frac{\tilde{U}_{\text {seen }, i}-U_{p, i}}{\tau_{p}}+g_{i}, \quad i=1,2,3
$$

It is assumed that the contribution of drag force and gravity dominates over the others (i.e., Basset force). Moreover, pressure forces are neglected because $\rho_{c}<<$ $\rho_{d}$.

In Eqs.(11) and (12) $\tau_{p}$ is the droplet relaxation time, calculated as:

$$
\tau_{p}=\frac{24 \rho_{d} d_{p}^{2}}{18 \mu_{c} C_{D} R e_{p}}
$$

where $C_{D}$ the drag coefficient given by the Schiller-Naumann semi-empirical correlation [17]:

$$
C_{D}= \begin{cases}\frac{24}{\operatorname{Re}_{p}}\left(1+0.15 R e_{p}^{0.687}\right), & R e_{p} \leq 1000 \\ 0.44, & R e_{p} \geq 1000\end{cases}
$$


The droplet Reynolds number reads:

$$
R e_{p}=\frac{\bar{\rho}_{c}\left|\tilde{U}_{\text {seen }}-U_{p}\right| d_{p}}{\mu_{c}}
$$

The Stokes number is defined as:

$$
S t=\frac{\tau_{p}}{\tau_{\eta}},
$$

where $\tau_{\eta}$ is the Kolmogorov time scale.

\section{Spray injection modeling and computational details}

\subsection{Spray injection modeling}

The transformation of the liquid jet leaving the nozzle orifice into primary atomized droplets up to the secondary atomization regime is not included in the present study. As such, the spray droplets are injected after completion of the secondary breakup regime.

The ConeInjection model available in OpenFOAM ${ }^{\circledR}$, version 2.4.0, is explained in detail as follows. The injection system is modeled as a point, located at the nozzle exit, at the center. The user specifies: the total mass to inject during the injection period $\left(t_{d}=1 s\right)$, the velocity magnitude for each parcel, $U_{m a g}$, the inner angle $\theta_{\text {in }}$, and the outer angle, $\theta_{\text {out }}$, the volume flow rate (which can be constant or not), $\dot{V}_{d}$, and the droplet size distribution.

The droplet size distribution and the values specified by the user are reported in Figure 3 and Table 2 respectively. The algorithm proceeds as follows:

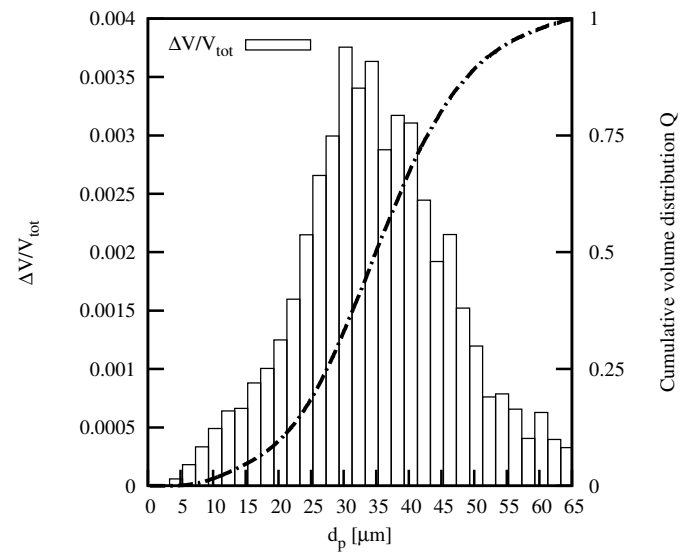

Fig. 3 Droplet size distribution defined in OpenFOAM ${ }^{\circledR 3}$ based on the experimental data [21]. Primary y axis: volume fraction per droplet size, $\Delta V / V_{t o t}$. Secondary y axis: Cumulative volume distribution $\mathrm{Q}$. 
Table 2 Values specified by the user in OpenFOAM ${ }^{\circledR}[26]$ for the spray injection

\begin{tabular}{l|lllll}
\hline & $\dot{m}_{d}[\mathrm{~g} / \mathrm{s}]$ & $\dot{V}_{d}\left[\mathrm{~m}^{3} / \mathrm{s}\right]$ & $\theta_{\text {in }}\left({ }^{\circ}\right)$ & $\theta_{\text {out }}\left({ }^{\circ}\right)$ & $U_{\text {mag }}[\mathrm{m} / \mathrm{s}]$ \\
\hline InjOF & 0.28 & $4.1 \cdot 10^{-7}$ & 20 & 60 & 30 \\
InjM & 0.28 & $4.1 \cdot 10^{-7}$ & - & - & 30 \\
\hline
\end{tabular}

1. Assign a velocity direction $U_{p, i}$ : For each parcel, the velocity direction is calculated by assigning an angle, selected through a random number generator function that takes an angle between the inner angle $\theta_{i n}$, and the outer angle, $\theta_{\text {out }}$.

2. Assign a diameter $d_{p}$ to each parcel: The parcel diameter is assigned based on the user defined droplet size distribution (Figure 3).

3. Define how many real droplets each parcel contains.

The assumption adopted here is that each parcel has the same initial mass according to:

$$
m_{p}=\frac{\dot{m}_{d}}{\dot{N}_{p}}
$$

with $\dot{N}_{p}$ the number of parcels injected per second. Therefore, assuming a spherical shape and knowing the liquid density, $\rho_{d}$; the $n_{p}$ is the number of real droplets inside each parcel.

$$
n_{p}=\frac{m_{p}}{\frac{\pi d_{p}^{3}}{6} \rho_{d}}
$$

It follows therefore that the number of droplets per parcel is inversely proportional to the droplet volume.

Step 1 in the algorithm above can be customized, replacing the random number generator function by an angle size distribution. A normal distribution with mean value equal to 40 (i.e., equal to the half nominal spray angle) and variance equal to 8 (to have a hollow cone spray between $24^{\circ}$ and $56^{\circ}$ ) has been implemented.

In the results section the original and modified injection methodology will be called 'InjOF' and 'InjM' respectively.

\subsection{Computational details}

The complex air co-flow injector geometry is replaced by an annular cylinder with $0.01 \mathrm{~m}$ length and inner and outer diameter equal to the annular outlet co-flow section as in the experiment (Figure 1). The remaining part of the computational domain, representative of the ambient, has a cylindrical shape. It has a diameter of $0.24 \mathrm{~m}$ (in order to ensure there is no interaction between the cylinder side wall and the spray jet), and is $0.25 \mathrm{~m}$ long ( to ensure no interference of the outlet BC). A schematic representation of the computational domain is reported in Figure 4. Four structured O-ring arrangement meshes are compared in this study and presented in Table 3. The top view of a mesh segment for the four grids is reported in Figure 5. CFD simulations have been performed using the reactingParcelFoam 

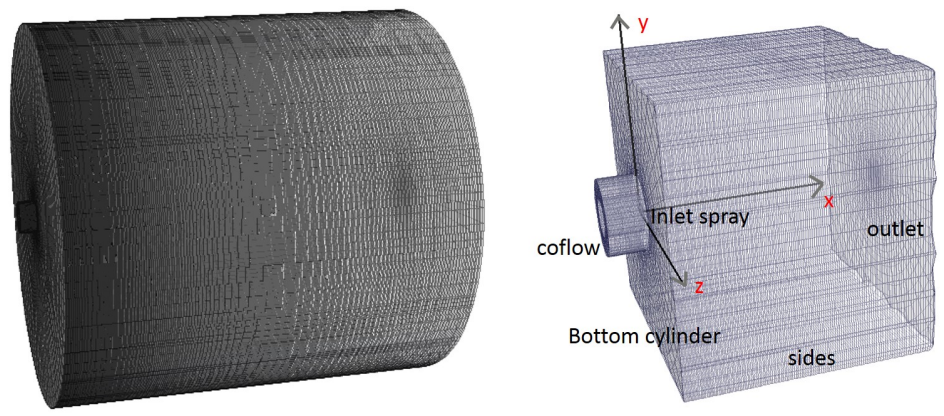

Fig. 4 Schematic representation of the computational domain (left) and mesh detail near the annular cylinder.
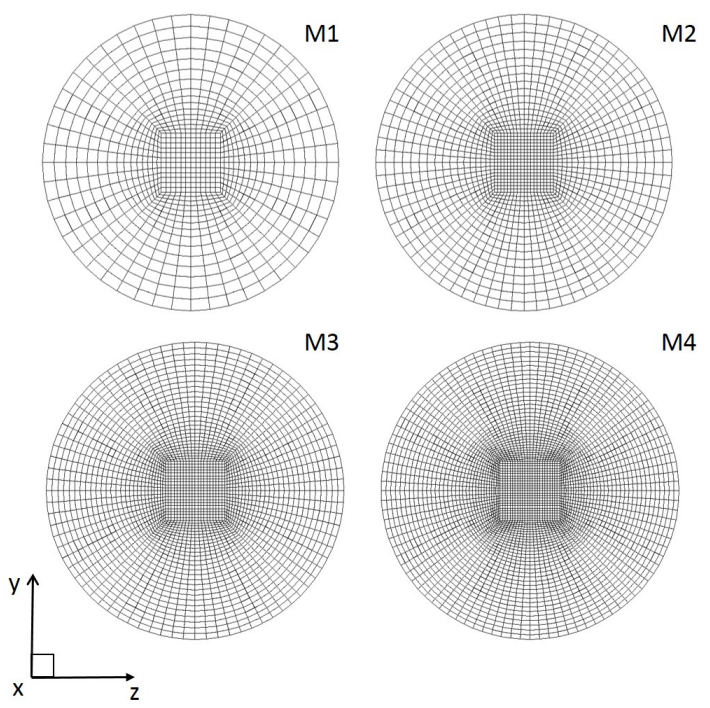

Fig. 5 Top view of a mesh segment for the four grids. From top left to bottom right: M1, M2, M3, M4.

solver in OpenFOAM-2.4.0 open source code [26]. This is a transient solver for compressible turbulent flows with a reacting, multiphase particle cloud. In the present study the reacting solver features have been disabled.

It is worth to mention that for the operating conditions at hand, an incompressible solver could be used. However, the compressible solver is applied in view of future numerical studies of the same case in reactive conditions. Velocity fluctuations in the annular co-flow were imposed using the Random Spot generator [9]. Using this $\mathrm{BC}$ at the inlet co-flow, the mean velocity profile, the integral length scale and 
Table 3 Minimum and maximum mesh size $[\mathrm{mm}]$ along the axial $\left(\delta_{a x}^{\min }, \delta_{a x}^{\max }\right)$ and radial $\left(\delta_{\text {rad }}^{\min }, \delta_{\text {rad }}^{\max }\right)$ direction.

\begin{tabular}{l|llll}
\hline & M1 & M2 & M3 & M4 \\
\hline$\delta_{a x}^{\min }$ & 0.34 & 0.25 & 0.20 & 0.17 \\
$\delta_{a x}^{\max }$ & 1.68 & 1.26 & 1.02 & 0.84 \\
$\delta_{\text {rad }}^{\min }$ & 0.34 & 0.22 & 0.20 & 0.16 \\
$\delta_{\text {rad }}^{\operatorname{mad}}$ & 11.8 & 7.5 & 6.7 & 6.3 \\
No.cells & $0.67 \cdot 10^{6}$ & $1.57 \cdot 10^{6}$ & $2.77 \cdot 10^{6}$ & $4.39 \cdot 10^{6}$ \\
\hline
\end{tabular}

Reynolds stress tensor should be provided. The mean velocity profile is assumed to follow the profile as measured in the experiments at the closest location from the nozzle and such that the mass flow rate is equal to the experimental operational conditions $\left(\dot{m}_{c}=6 \mathrm{~g} / \mathrm{s}\right)$. The integral length scale is set equal to $d_{h}=0.01 \mathrm{~m}$. The Reynolds stress tensor profile accounts only for the normal components and they are chosen uniform and equal to the average values available from the experiments at the closest axial location from the nozzle.

The overview of the BCs for the velocity and pressure as implemented in OpenFOAM ${ }^{\circledR}$ [26], is provided in Table 4.

The sets of discretization schemes have been summarized in Table 5

Table 4 Boundary conditions implemented in OpenFOAM ${ }^{\circledR}$.

\begin{tabular}{l|ll}
\hline & Velocity & Pressure \\
\hline inletspray & zeroGradient & zeroGradient \\
coflow & Random Spots method & zeroGradient \\
bottom cylinder & FixedValue=0 & zeroGradient \\
sides & pressureInletOutletVelocity & totalPressure \\
outlet & zeroGradient & zeroGradient \\
\hline
\end{tabular}

Table 5 Discretization schemes.

\begin{tabular}{l|ll}
\hline Calculation & Keyword & Scheme \\
\hline Time derivative & timeScheme & Euler \\
Gradient terms & gradSchemes & Gauss Linear \\
Convective terms & $\operatorname{div}($ phi,U) & Gauss upwind \\
Convective terms & $\operatorname{div}((\operatorname{muEff}$ dev2 $(\mathrm{T}(\operatorname{grad}(\mathrm{U}))))$ & Gauss Linear \\
Diffusive terms & laplacianSchemes & Gauss linear corrected \\
\hline
\end{tabular}

The choice of a first order scheme for the velocity convective term was made because the current work investigates the numerical results of 17 simulations and robustness was preferred over accuracy. The 'only gas' cases have also been simulated with a $2^{\text {nd }}$ order scheme (Gauss linear) and while somewhat faster converging of the jet is observed, due to the less dissipative nature of $2^{n d}$ order schemes than 
$1^{s t}$ order schemes, the trend with respect to the grid sensitivity remains unchanged (i.e., the results on M1 and M2 are similar, and the ones on M3 and M4 are similar, too). Therefore, the present analysis is deemed representative for higher order schemes as well. As described in the paragraph on the injection methodology, the number of droplets per parcel is inversely proportional to the droplet volume. The number of droplets per parcel as function of the droplet diameter using $\dot{N}_{p}=0.5 \cdot 10^{6}, 2.2 \cdot 10^{6}$ and $4 \cdot 10^{6}$ parcels $/ s$ is shown in Figure 6 . The graph shows a sharp decrease in the number of droplets per parcel when the droplet diameter increases. Droplets with diameter below $d_{p}<10 \mu \mathrm{m}$ are the most clustered: the smallest parcel can contain from $10^{3}$ droplets, in case of $4 \cdot 10^{6}$ parcels $/ \mathrm{s}$, up to $10^{5}$ droplets in case of $0.5 \cdot 10^{6}$ parcels $/ \mathrm{s} . \dot{N}_{p}=2.2 \cdot 10^{6}$ parcels $/ \mathrm{s}$ is chosen according to [12], whose approach is to secure that all the droplets are represented by at least one parcel. This can be done by dividing the total mass flow rate $(0.28 \mathrm{~g} / \mathrm{s})$ by the mass of the biggest droplet $\left(d_{\max }=65 \mu \mathrm{m}\right)$, leading to a number of $2.2 \cdot 10^{6}$ parcels $/ \mathrm{s}$. The other two values are used to investigate if more $\left(0.5 \cdot 10^{6}\right.$ parcles $\left./ \mathrm{s}\right)$ or less $\left(4 \cdot 10^{6}\right.$ parcles $\left./ \mathrm{s}\right)$ clustering has any impact on the numerical results. The Eulerian-Lagrangian coupling approach has been referred as the semi unsteady approach [27]. In this approach the time step is controlled by the gas phase (Eulerian time step). The Lagrangian time step is smaller and controlled by the smallest time scale of the phenomena involved (i.e., particle response time, grid size, liquid Courant number). Therefore, to cover the Eulerian step timespan, multiple Lagrangian time steps are needed. During these time steps the flow field used for the integration of the Lagrangian equations does not change. Solving the velocity and pressure equations with the PISO algorithm, the liquid and gas Courant number have been chosen equal to 0.3. However, for M1, when $\dot{N}_{p}=0.5 \cdot 10^{6}$ parcels $/ \mathrm{s}$ is used, the Courant number had to be lowered to 0.2 due to instabilities in the pressure-velocity loop, which eventually caused divergence.

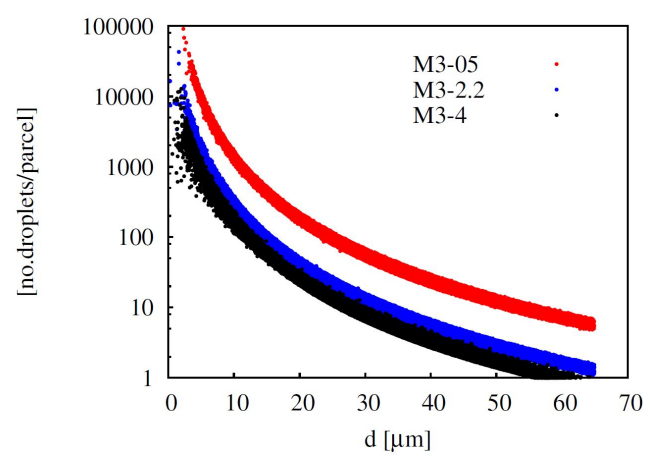

Fig. 6 Number of droplets per parcel as function of the droplet diameter. Red: $\dot{N}_{p}=0.5 \cdot 10^{6}$ (M3-05), Blue: $\dot{N}_{p}=2.2 \cdot 10^{6}(\mathrm{M} 3-2.2)$, Black: $\dot{N}_{p}=4 \cdot 10^{6}$ parcels $/ \mathrm{s}(\mathrm{M} 3-4)$. 
4.3 Cases: summary and computational time

The wall-clock time to compute $1 s$ simulation time varies both with the mesh size and $\dot{N}_{p}$. The time span to cover $1 s$ simulation is reported for each case in Table 6.

M3 and M4 can be executed only on a High Performance Computing (HPC) infrastructure with a performant InfiniBand network. These calculations have been executed on the UGent Tier-2 cluster (2x 12-core Intel E5-2680v3 (Haswell-EP @ $2.5 \mathrm{GHz})$ ) and on the Tier-1 cluster BrENIAC (2x 14-core Xeon E5-2680v4 (Broadwell @ 2.4GHz)). Each case has a different number of processors, chosen after a node scalability test, aimed to maximize both speed-up and efficiency of the calculations.

Table 6 Cases performed for grid-parcels convergence. The simulation time (days) is referred to time needed to perform $1 s$ simulation for each case.

\begin{tabular}{l|l|l|l|l|l}
\hline & $\begin{array}{l}\text { Case } \\
\text { (label) }\end{array}$ & $\begin{array}{l}\text { M1 } \\
{[\text { days }]}\end{array}$ & $\begin{array}{l}\text { M2 } \\
{[\text { days }]}\end{array}$ & $\begin{array}{l}\text { M3 } \\
{[\text { days }]}\end{array}$ & $\begin{array}{l}\text { M4 } \\
{[\text { days }]}\end{array}$ \\
\hline Gas only & M -OG & 1.6 & 3.1 & 3.4 & 5.5 \\
$0.5 \cdot 10^{6}$ parcels $/ s$ & M -05 & 3.3 & 8.9 & 11.7 & 14.1 \\
$2.2 \cdot 10^{6}$ parcels $/ s$ & M -2.2 & 6.8 & 9.5 & 17.2 & 19.6 \\
$4.0 \cdot 10^{6}$ parcels $/ s$ & M -4 & 14.3 & 19.8 & 35.7 & 38.5 \\
\hline HPC (no.proc) & & Tier-2, 24 & Tier-2, 96 & Tier-1, 140 & Tier-1, 168 \\
\hline
\end{tabular}




\section{Results}

In section 5.1 the grid convergence study is performed analyzing first the LES calculations without liquid spray (cases labeled as OG, 'only gas'). Here, the main objective is the identification of the most suitable mesh of the four grids examined, based on level of description and computational costs.

Further, in section 5.2 the simulations performed with the chosen grid and including the spray (cases labeled as LG), are discussed. Particularly, a threshold for the droplet diameter will be determined to distinguish among droplets which are completely dragged by the gas flow field and the ones which pursue their motion. Comparison between experiments and simulations is the objective of section 5.3, with the aim of validating the numerical framework. All the results in section 5.25.3 are obtained with InjM. In section 5.4 this choice is justified via comparison with numerical results obtained using InjOF (i.e, the existing model as available in OpenFOAM $\left.{ }^{\circledR}\right)$.

\subsection{Grid sensitivity study}

Figure 7 provides Favre mean averaged axial velocity contours plots on the four different computational grids. Time averaging starts after $0.05 s$ and statistics are collected over a period of $0.95 \mathrm{~s}$. The positions of the measured profiles at $\mathrm{x}$ $\in[0.007,0.01,0.013,0.016,0.02,0.025,0.03,0.04,0.05 \mathrm{~m}]$ are indicated in the figure as well.

The jet approaching the exit of the annular duct does not undergo substantial changes until $x=0.01 \mathrm{~m}$. Differences in this region, between the four grids, are observed for the maximum velocity value at the potential core, which is higher for M3 and M4.

From $x=0.01 m$ onward, the jet merges towards the central axis. In this region higher velocities around the center are observed for M3 and M4, which show similar behavior.

The profiles of the Favre mean averaged axial velocities at different axial positions are presented in Figure 8 . In the near nozzle region, $(x=0.007 \mathrm{~m})$ the velocity magnitude up in the recirculation region is higher for cases M3 and M4 due to the aforementioned higher velocities at the nozzle exit. Besides, the re-circulation region extends longer for M3 and M4 than for M1 and M2.

Due to the longer re-circulation on M3 and M4, at $x=0.01 m$ the velocity at the center is lower.

From $x=0.013 m$ onward, the profiles for M3 and M4 almost perfectly coincide. From $x=0.016 m$ to $x=0.03 m$ velocities at the center are higher for M3 and M4 and the jet tends to be slightly wider for M1 and M2. From $x=0.04 m$ onward, i.e., after about 4 annular nozzle diameters, the jet expands like a single jet. In line with the above, the velocity values in the central region are lower with M1 and M2 than with M3 and M4.

The influence of the mesh size on the subgrid-scale eddy viscosity level is illustrated in Figure 9 at the same positions.

Unlike the velocity profiles, the $\mu_{s g s} / \mu$ plots are different for each case, shifting to lower values from M1 to M4. M1 and M2 give a higher eddy viscosity in the region close to the nozzle, especially at the potential jet core. Resolving more energy- 

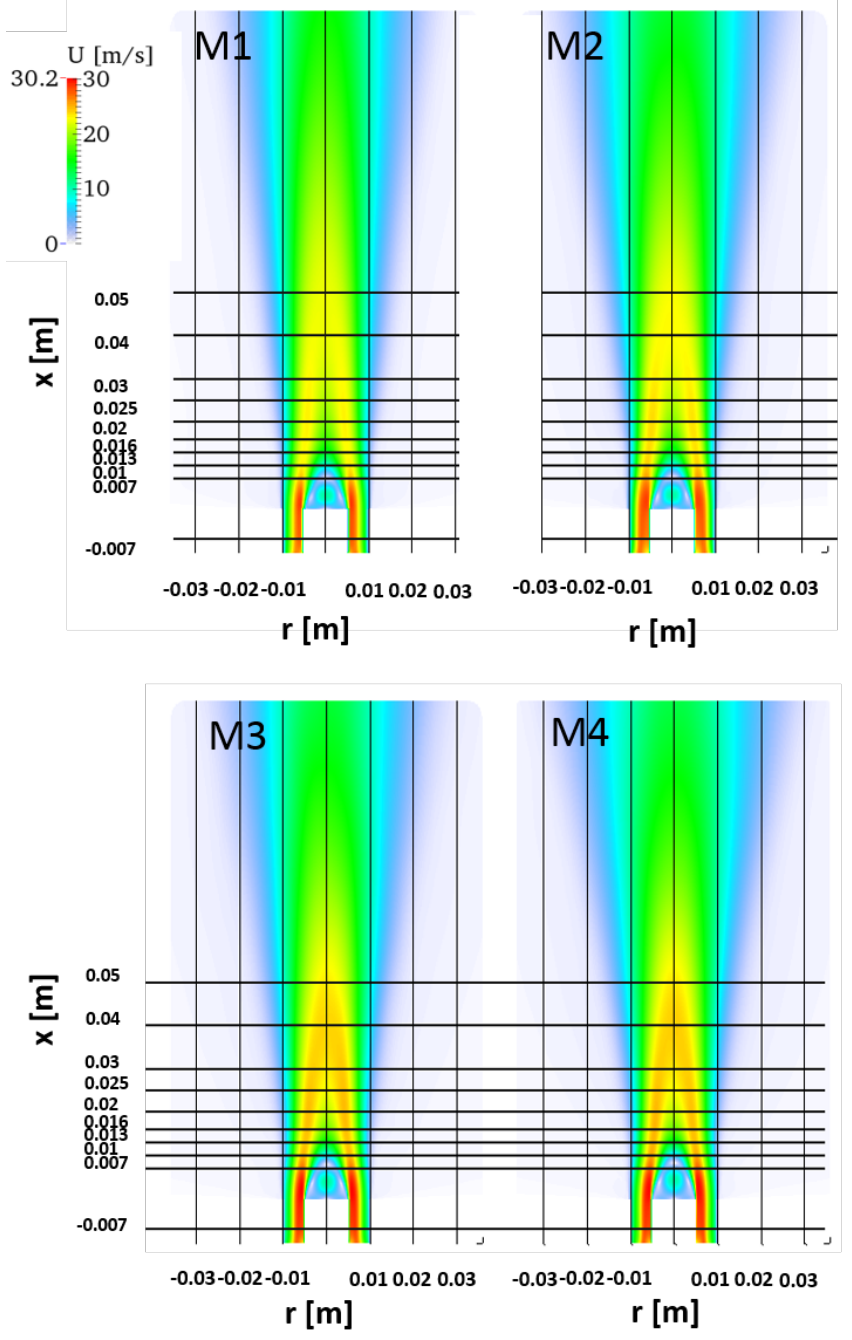

Fig. 7 Contour plots of the time averaged Favre mean velocity magnitude. From top left to bottom right: M1, M2, M3, M4 (case with only gas, OG). Time averaging period is $0.95 \mathrm{~s}$.

containing motions induces drastic changes to the estimated velocities from M2 to M3, while the further refinement from M3 to M4 does not have a significant impact. The profiles obtained with M1 and M2 differ, whereas those obtained with M3 and M4 are very close, which suggests grid insensitivity when the ratio $\mu_{s g s} / \mu<0.2$ (Figure 9). An analysis of the energy spectra in points of the domain located in the region of interest, reveals that for mesh M3 and M4 the slope of $-5 / 3$, which characterises the inertial subrange, is well captured (not shown here). These findings suggest grid insensitivity using a streamwise mesh spacing $\delta_{a x} \leq 0.2 \mathrm{~mm}$ and spanwise mesh spacing $\delta_{\text {rad }} \leq 0.2 \mathrm{~mm}$ (Table 3). In other words, both M3 and M4 represent reliable grids to perform the simulations at 

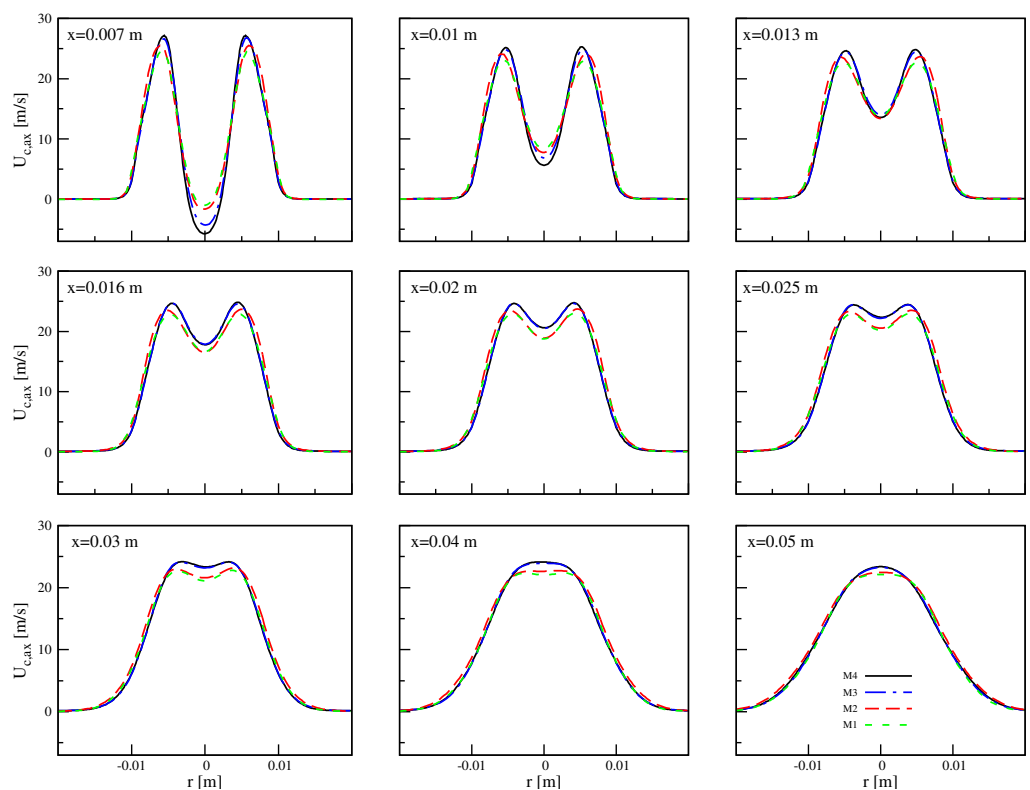

Fig. 8 Time-averaged spanwise profiles of the axial mean velocity, $U_{c, a x}[\mathrm{~m} / \mathrm{s}]$, on grids M1 (short-dashed green), M2 (long-dashed red), M3 (dash-dotted blue), M4 (solid black). Cases with only gas phase, OG. Time averaging period is $0.95 \mathrm{~s}$.
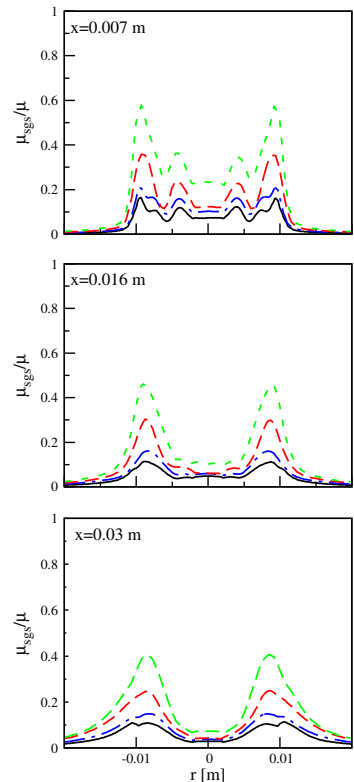
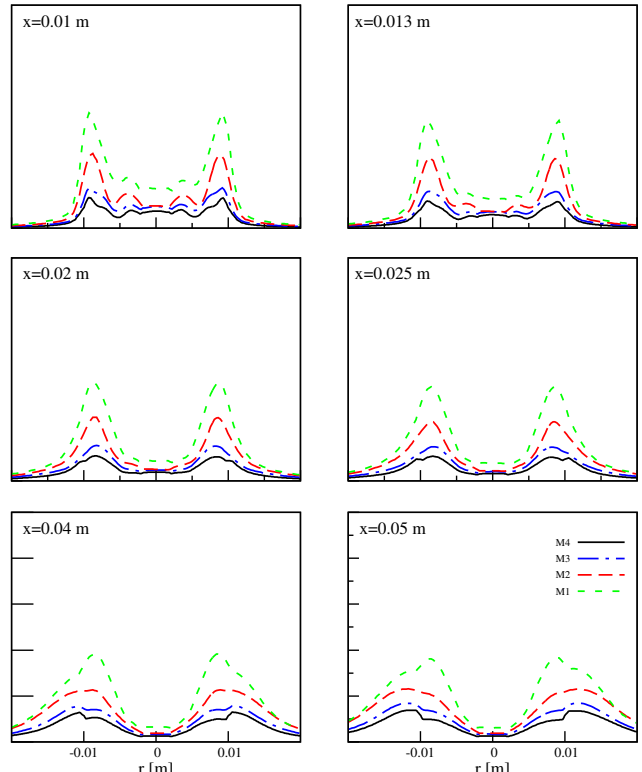

Fig. 9 Time-averaged spanwise profiles of the ratio of the subgrid-scale viscosity to the laminar viscosity, $\mu_{s g s} / \mu$ on grids M1 (short-dashed green), M2 (long-dashed red), M3 (dash-dotted blue), M4 (solid black). Cases with only gas phase, OG. Time averaging period is $0.95 \mathrm{~s}$. 
hand. Therefore, the differences in calculation time and number of processors to be used, which are reported in Table 3 for $1 s$ simulation, led to the choice of running the two-phase cases on grid M3. Nevertheless, some discussion of the results with both liquid and gas phase will be done considering the four grids to highlight how a poor mesh resolution can affect the Lagrangian phase results as well.

\subsection{Gas-Liquid Phase}

The mutual interaction between gas and liquid droplets can have an impact on the gaseous flow field. In order to assess this, the results on M1, M2, M3, M4 with only gas (OG) and both gas and liquid (LG) are compared at $x \in[0.02 \mathrm{~m}, 0.03 \mathrm{~m}, 0.04 \mathrm{~m}]$. The results are presented as velocity offset, i.e., difference between the mean axial velocities of OG and LG. The comparison is done for different numbers of parcels per second $\dot{N}_{p}$. From the results provided in Figure 10, it can be observed that:

- The highest offset value $(\sim 1 \mathrm{~m} / \mathrm{s})$ is at the centerline.

- The offset does not strongly depend on the mesh size. It decreases along the axis, because the momentum transfer between liquid and gas becomes smaller.

- The offset decreases if $\dot{N}_{p}$ is held constant and the mesh is refined for $\dot{N}_{p}=$ $0.5 \cdot 10^{6}$ parcels $/ \mathrm{s}$. As reported in the literature, [19], [18], [7] the parcels number cannot be fixed if the mesh is refined; this would amplify the statistical error, the latter defined as [6]:

$$
\Sigma_{F}=\frac{c_{F} \theta}{\sqrt{\dot{N}_{p} / M}}
$$

where $c_{F}$ is a statistical error coefficient, $\theta$ is a standardized normal random variable and $M=M_{x}+M_{y}+M_{z}$ is the total number of grid cells. Therefore, using the same grid, the statistical error decreases as the number of computational particles per cell $N_{p c}$ increases, reaching the limit of 0 if the ratio between particles/parcels is one. Estimation of the error requires multiple identical simulations to calculate the coefficient $c_{F}$. This was considered beyond the scope of the present work . In this case the error could be linked to the absence of enough parcels at the center that in turn would reduce the impact from the liquid to the gas phase. The offset values with $\dot{N}_{p}=2.2 \cdot 10^{6}$ parcels $/ \mathrm{s}$ and $\dot{N}_{p}=4 \cdot 10^{6}$ parcels $/ \mathrm{s}$ are very close for all the grids, which suggests "parcels convergence" for $\dot{N}_{p} \geq 2.2 \cdot 10^{6}$ parcels $/ s$.

The parcels' behavior along the axial coordinate can be explained analyzing the particle Stokes number (Eq.(16)).

To post-process the parcels' Stokes number, a single step of the spray cloud and the gas flow field is saved. Knowing the cell indexes at which the parcels are temporarily located, it is possible to calculate the Kolmogorov time scale at the corresponding cell center and the particle response time, based on the particle Reynolds number, $R e_{p}$, and the physical properties of both the carrier and liquid phase.

Three different regions have been examined: $x \in[0.005-0.007 \mathrm{~m}], x \in[0.016-$ $0.02 \mathrm{~m}]$ and $x \in[0.04-0.05 \mathrm{~m}]$, representative of the near-nozzle, intermediate and far downstream region respectively (locations shown in Figure 7 ). The results are reported in Figures 11-13 using M3, InjM and $\dot{N}_{p}=2.2 \cdot 10^{6}$ parcels $/$ s. 

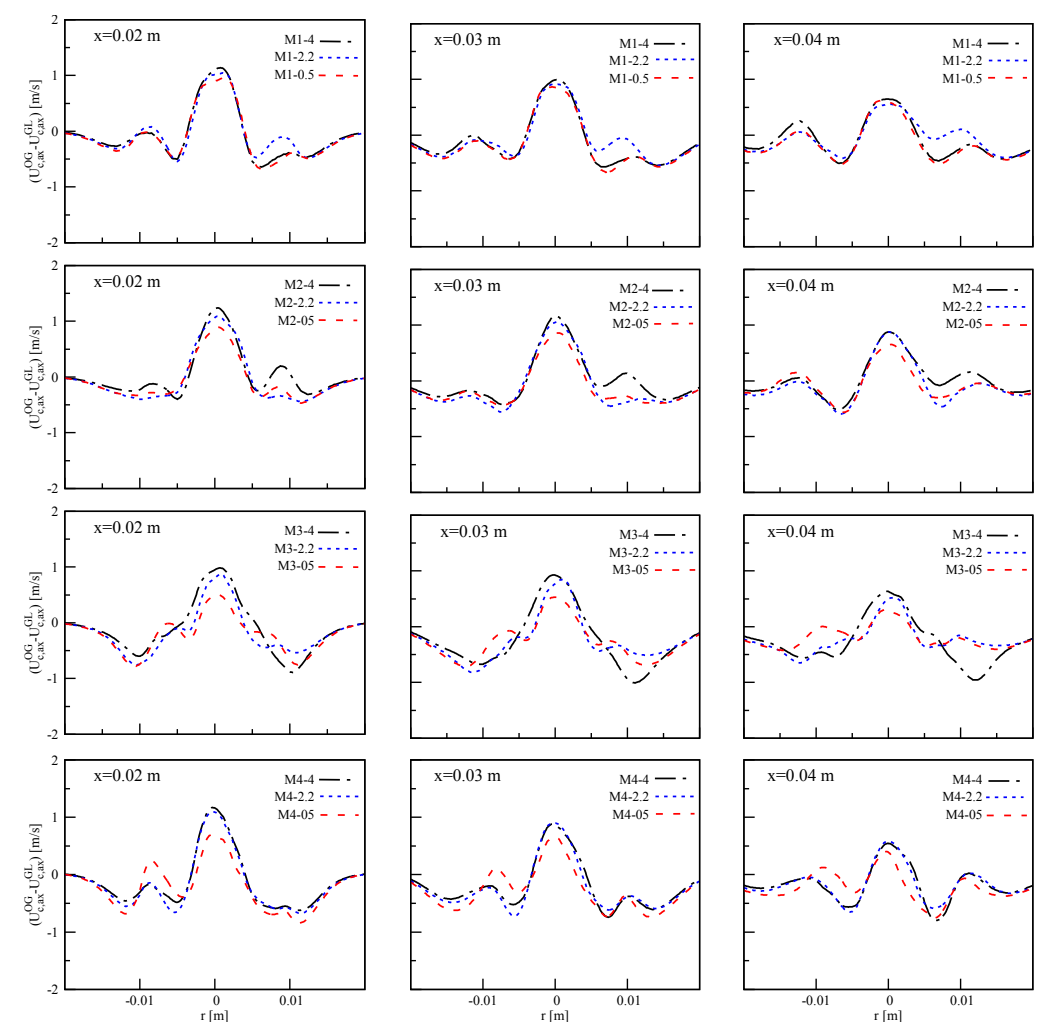

Fig. 10 Difference between the mean axial velocities of OG and LG. From top to bottom: M1, M2, M3, M4. From left to right: $x=0.02 m, x=0.03 m, x=0.04 \mathrm{~m}$. black lines: $N p=4 \cdot 10^{6}$ parcels $/ \mathrm{s}$; blue lines: $N p=2.2 \cdot 10^{6}$ parcels $/ \mathrm{s}$; red lines: $N p=0.5 \cdot 10^{6}$ parcels $/ \mathrm{s}$. Time averaging period is $0.95 \mathrm{~s}$.

It has to be mentioned, though, that, as there is no dispersion model for subgrid scale contributions, the droplets are influenced only by the resolved flow field and the Kolmogorov time scale is not the most relevant one to assess whether or not droplets follow the flow field. Nevertheless the Kolomogorov time scale can be used as reference here because the current simulation is highly resolved, as indicated by the small ratio of eddy viscosity and laminar viscosity in Figure 9 . In the nearnozzle region the imposed spray initial conditions control the entire spray cloud, except for class $d_{p} \in[0-15 \mu \mathrm{m}]$. As reported in Figure11-a, for this class, the Stokes number is below 1 as the particle relaxation time is always smaller than the Kolmogorov time scale.

Droplets with $d_{p}>15 \mu \mathrm{m}$ have a particle relaxation time higher than the Kolmogorov time scale for $20^{\circ}<\theta<40^{\circ}$ (Figure 11-c, 11-d). These droplets pass straight through the jet potential core. For $\theta>40^{\circ}$ time and length scales increase (low turbulence level) and the $S t$ decreases for all the droplet sizes (Figure 11-b).

In the intermediate region $(x \in[0.016-0.02 \mathrm{~m}])$ there is an overall decrease in particle $S t$ number from a maximum value of $S t=25$ (Figure 11a) to $S t=10$ 

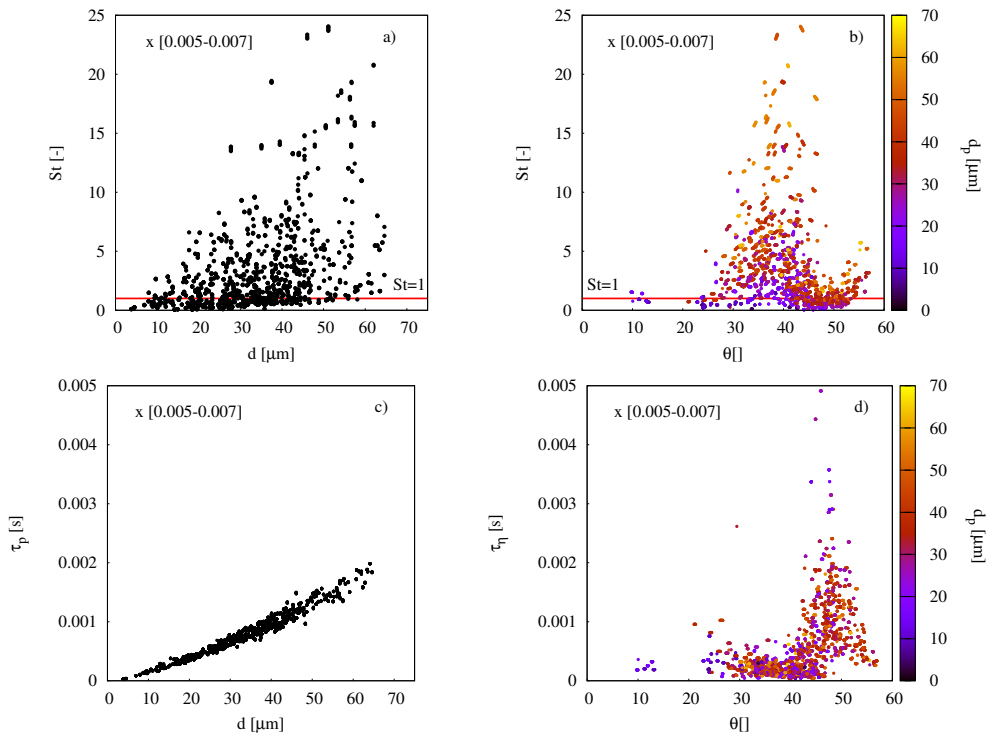

Fig. 11 a) Stokes number, $S t$ plotted versus droplet diameter, $d_{p}$. b) Stokes number, $S t$ vs half spray angle, $\theta$. Parcels are colored based on the droplet diameter, $d_{p}[\mu m]$. c) particle response time, $\tau_{p}$ versus droplet diameter, $d_{p}$. d) Kolmogorov time scale, $\tau_{\eta}$ along the radius $r$. Parcels are colored based on droplet diameter, $d_{p}[\mu \mathrm{m}]$. Results at $x \in[0.005 \mathrm{~m}-0.007 \mathrm{~m}]$. Case M3, $\dot{N}_{p}=2.2 \cdot 10^{6}$ parcels $/ \mathrm{s}$ and InjM.

(Figure 12a). Droplets with $d_{p}<30 \mu m$ tend to concentrate to the left of the gaseous shear layer, which is located at $\theta \sim 25^{\circ}$; vice versa for $d_{p}>30 \mu \mathrm{m}$ (Figure $12-\mathrm{b})$. Whereas in the near-nozzle region the spray is bounded between the imposed inner and outer spray angles $20^{\circ}<\theta<60^{\circ}$, in the intermediate region the small droplets are dragged away by the gas phase and distributed in the center (Figure 12-b). In the far downstream region $(x \in[0.04-0.05 \mathrm{~m}])$, the particle $S t$ number decreases further (Figure 13). At this location almost all the parcels have lost the history of the imposed injection boundary conditions and they mostly follow the gas phase. The majority of the parcels with $d_{p} \in[20-40 \mu m]$ tend to be concentrated close to the shear layer, located at $\theta \sim 12^{\circ}$ here (Figure 13-b).

The increase of $\dot{N} p$ from $2.2 \cdot 10^{6}$ parcels $/ s$ to $4 \cdot 10^{6}$ parcels $/ s$ doubles the computational time, as reported in Table 6 , without inducing any beneficial impact in the analysis of the graphs in Figure 11-13.

The evidence presented in this section suggests that $\dot{N}_{p}$ must be adequately chosen according to the mesh size. The choice of M3 in section 5.1 led to the choice of using $N p \geq 2.2 \cdot 10^{6}$ parcels $/ s$ for LG calculations. The analysis of the experimental data, therefore, will be done using M3 with $N p=2.2 \cdot 10^{6}$ parcels $/ \mathrm{s}$. Besides, the data in Figures 11-13 reveal that parcels with $d_{p}<15 \mu \mathrm{m}$ completely follow the gas phase $(S t<1)$. This threshold will be used to discuss results in Section 5.3 .

The findings from Figures 11-13 suggest that the gaseous shear layer can be viewed, from the intermediate region onward, as the spray inner edge. Because the position of the shear layer is sensitive to the mesh grid, this will vary according to the cho- 

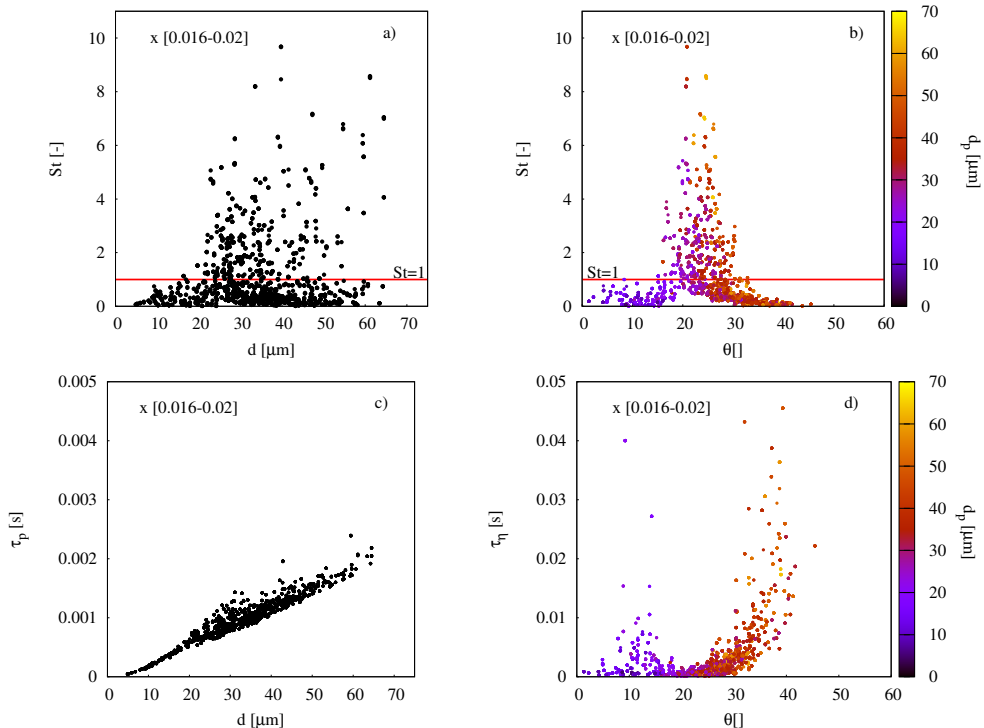

Fig. 12 a) Stokes number, $S t$ plotted versus droplet diameter, $d_{p}$. b) Stokes number, $S t$ vs half spray angle, $\theta$. parcels are colored based on the droplet diameter, $d_{p}[\mu \mathrm{m}]$. c) particle response time, $\tau_{p}$ versus droplet diameter, $d_{p}$. d) Kolmogorov time scale, $\tau_{\eta}$ along the radius $r$. Parcels are colored based on droplet diameter $\left(d_{p}[\mu m]\right)$. Results at $x \in[0.016 m-0.02 m]$. Case M3, $\dot{N}_{p}=2.2 \cdot 10^{6}$ parcels $/ s$ and InjM.
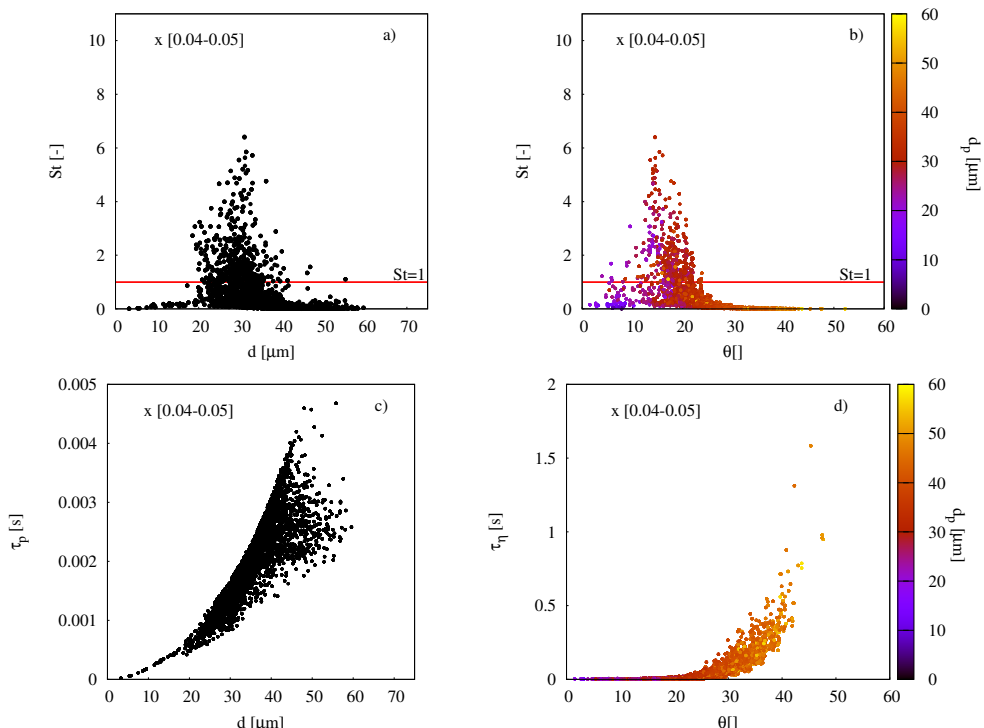

Fig. 13 a) Stokes number, $S t$ plotted versus droplet diameter, $d_{p}$. b) Stokes number, $S t$ vs half spray angle, $\theta$. parcels are colored based on the droplet diameter, $d_{p} . \mathbf{c}$ ) particle response time, $\tau_{p}$ versus droplet diameter, $d_{p}[\mu m]$. d) Kolmogorov time scale, $\tau_{\eta}$ along the radius $r$. Parcels are colored based on droplet diameter $d_{p}[\mu \mathrm{m}]$. Results at $x \in[0.04 m-0.05 m]$. Case M3, $\dot{N}_{p}=2.2 \cdot 10^{6}$ parcels $/ \mathrm{s}$ and InjM. 

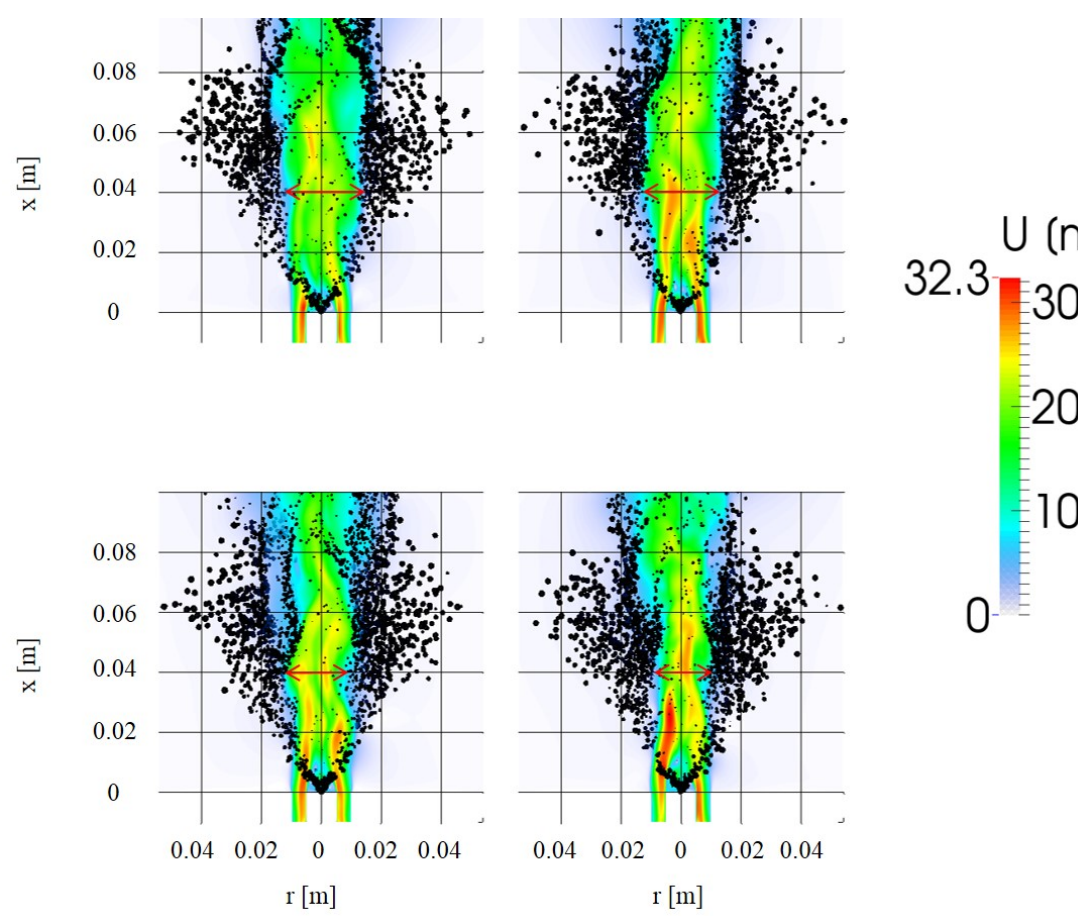

Fig. 14 Instantaneous velocity contour plots together with spray parcels (represented with dots having size according to their diameter). From top left to bottom right: M1, M2, M3 and M4. (InjM with $2.2 \cdot 10^{6} \mathrm{parcel} / \mathrm{s}$ ). 


\subsection{Comparison to experimental data}

Numerical spanwise profiles of the Favre mean axial gas velocity and mean axial liquid velocity are compared to experimental data and presented in Figure 15.
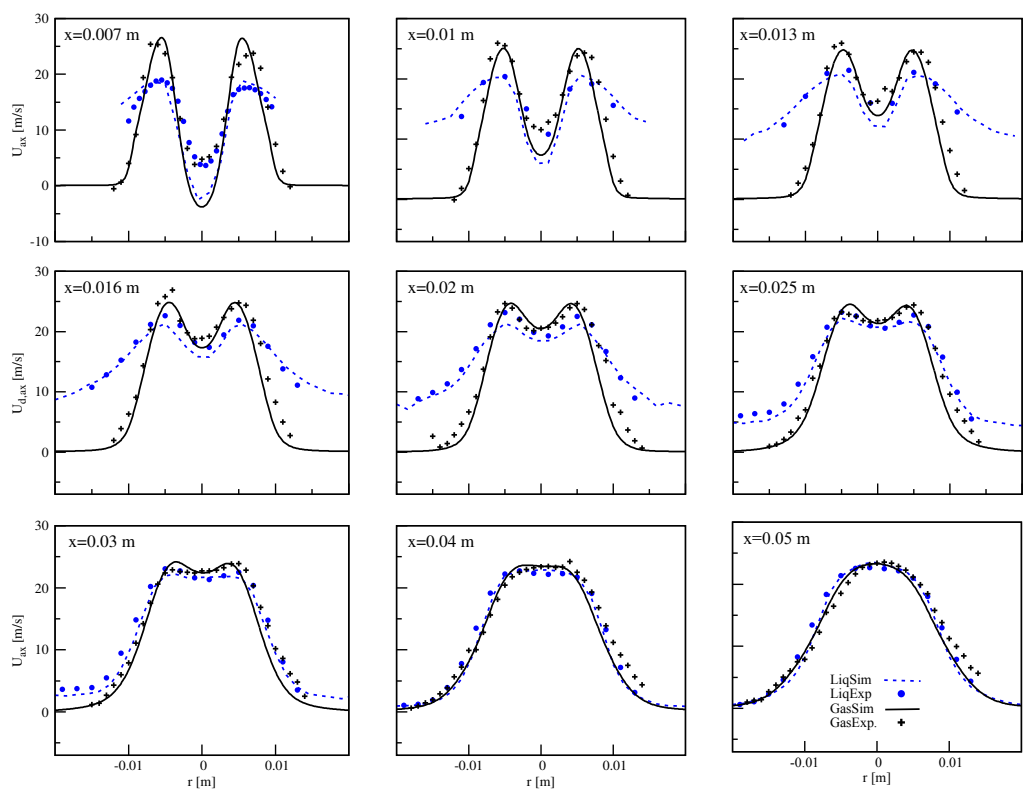

Fig. 15 Mean gas axial velocity (black crosses and lines) together with mean liquid axial velocity (blue dot and dashed line) plotted versus the radius $\mathrm{r}[\mathrm{m}]$ at different axial positions $\mathrm{x}[\mathrm{m}]$ (grid M3 with InjM and $2.2 \cdot 10^{6}$ parcels/s). Time averaging period is $0.95 \mathrm{~s}$.

The main discrepancies between experimental and numerical results for the gas phase are observed from $x=0.007 \mathrm{~m}$ up to $x=0.013 \mathrm{~m}$. Neither the exact location of the end of the re-circulation region $(x=0.007 \mathrm{~m})$, nor the velocity magnitude are correctly predicted in the simulations. These discrepancies are likely to be related to the simplification of complex injector geometry as an annular cylinder, or to the imposed artificial turbulence at the inlet, or to both. This is considered future research for the time being. From $x=0.016 \mathrm{~m}$ onward, differences between experiments and numerical results at the center are below 5\%. An error analysis of the maximum axial velocity values for the gas phase along the axial coordinate is presented in Table 7 .

From the data it can be observed that the two off-center peaks are correctly predicted at all the locations with the exception of $x=0.007 \mathrm{~m}$ ( $11.4 \%$ error).

The above mentioned discrepancies for the gas phase velocity induce an error in the liquid phase prediction as well. This error is higher at the location where the $S t<1$ because the droplets follow the gas phase motion. As reported in Figures 11-13 this happens for $d_{p}<15 \mu m$, which tend to be distributed preferentially in the center. Subsequently, similarly to the gas phase, simulations results differ from experiments at $x=0.007 \mathrm{~m}, x=0.01 \mathrm{~m}$ and $x=0.013 \mathrm{~m}$ in the center (Figure 15). On the contrary, at the outer branch, from the jet potential core to 
Table 7 Error analysis of the maximum mean axial velocity for the gas phase at different axial locations.

\begin{tabular}{l|lll}
\hline $\mathbf{x}[\mathbf{m}]$ & $U_{\text {exp }}^{a x}[\mathrm{~m} / \mathrm{s}]$ & $U_{\text {sim }}^{a x}[\mathrm{~m} / \mathrm{s}]$ & $\operatorname{Err}(\%)$ \\
\hline 0.007 & 23.76 & 26.50 & 11.4 \\
0.01 & 24.32 & 24.99 & 2.75 \\
0.02 & 24.65 & 24.67 & 0.08 \\
0.03 & 23.90 & 23.92 & 0.08 \\
0.04 & 23.38 & 23.48 & 0.42 \\
0.05 & 23.38 & 23.31 & 0.29 \\
\hline
\end{tabular}

the outer radius, the agreement is good. In fact, as reported in Figure 11, the droplets with $d_{p}>15 \mu \mathrm{m}$ follow their ballistic motion defined by the injection boundary conditions.

From $x=0.016 m$ onward, the gap between experiments and simulations, similarly to the gas phase, reduces and the agreement between experiments and simulations is satisfactory. An error analysis of the maximum axial velocity values for the liquid phase along the axial coordinate is presented in Table 8. The maximum error is at $x=0.02 \mathrm{~m}$. The comparison between simulations and experiments for

Table 8 Error analysis of the maximum mean axial velocity for the liquid phase at different axial locations.

\begin{tabular}{l|lll}
\hline $\mathbf{x}[\mathbf{m}]$ & $U_{\text {exp }}^{a x}[\mathrm{~m} / \mathrm{s}]$ & $U_{\text {sim }}^{a x}[\mathrm{~m} / \mathrm{s}]$ & $\mathbf{\operatorname { E r r } ( \boldsymbol { \% } )}$ \\
\hline $0.007 \mathrm{~m}$ & 19.04 & 18.78 & 1.36 \\
$0.01 \mathrm{~m}$ & 20.47 & 20.18 & 1.42 \\
$0.02 \mathrm{~m}$ & 23.16 & 21.66 & 6.47 \\
$0.03 \mathrm{~m}$ & 23.16 & 21.86 & 5.94 \\
$0.04 \mathrm{~m}$ & 22.19 & 22.43 & 1.09 \\
$0.05 \mathrm{~m}$ & 22.57 & 22.94 & 1.64 \\
\hline
\end{tabular}

the liquid mean axial velocity per droplet class size is shown in Figure 16. At $x=0.007 \mathrm{~m}$ results from experiments and simulations are different both for $d_{p} \in$ $[0-10 \mu \mathrm{m}]$ and $d_{p} \in[10-20 \mu \mathrm{m}]$. These discrepancies are directly linked to those observed for the gas phase at the same location, confirming the findings discussed in Figure 11-a. Better agreement between experiments and simulations is observed for $d_{p} \in[20-30 \mu \mathrm{m}]$ and $d_{p} \in[30-40 \mu \mathrm{m}]$. For $d_{p} \in[40-50 \mu \mathrm{m}]$, the velocity is over-predicted, showing the limitation of the injection model by fixing a velocity magnitude value for all the parcels. Finally it is possible to notice that these three classes have statistics missing in the center. Two possible interpretations are:

- In the experiments statistics are collected during a time-span of $4 s$, versus $1 s$ in the calculations.

- The strict separation in the shear layer between $d_{p}>30 \mu m$ and $d_{p}<30 \mu m$ (Figure 12-b) is less severe in reality.

Using $\dot{N}_{p}=4 \cdot 10^{6} \mathrm{parcel} / \mathrm{s}$ slightly improves the results. This is shown only for the $d_{p} \in[20-30 \mu \mathrm{m}]$ at $x=0.007 \mathrm{~m}$, where differences can be noticed.

At $x=0.02 m$ the agreement is good for all the droplet size classes, except for 

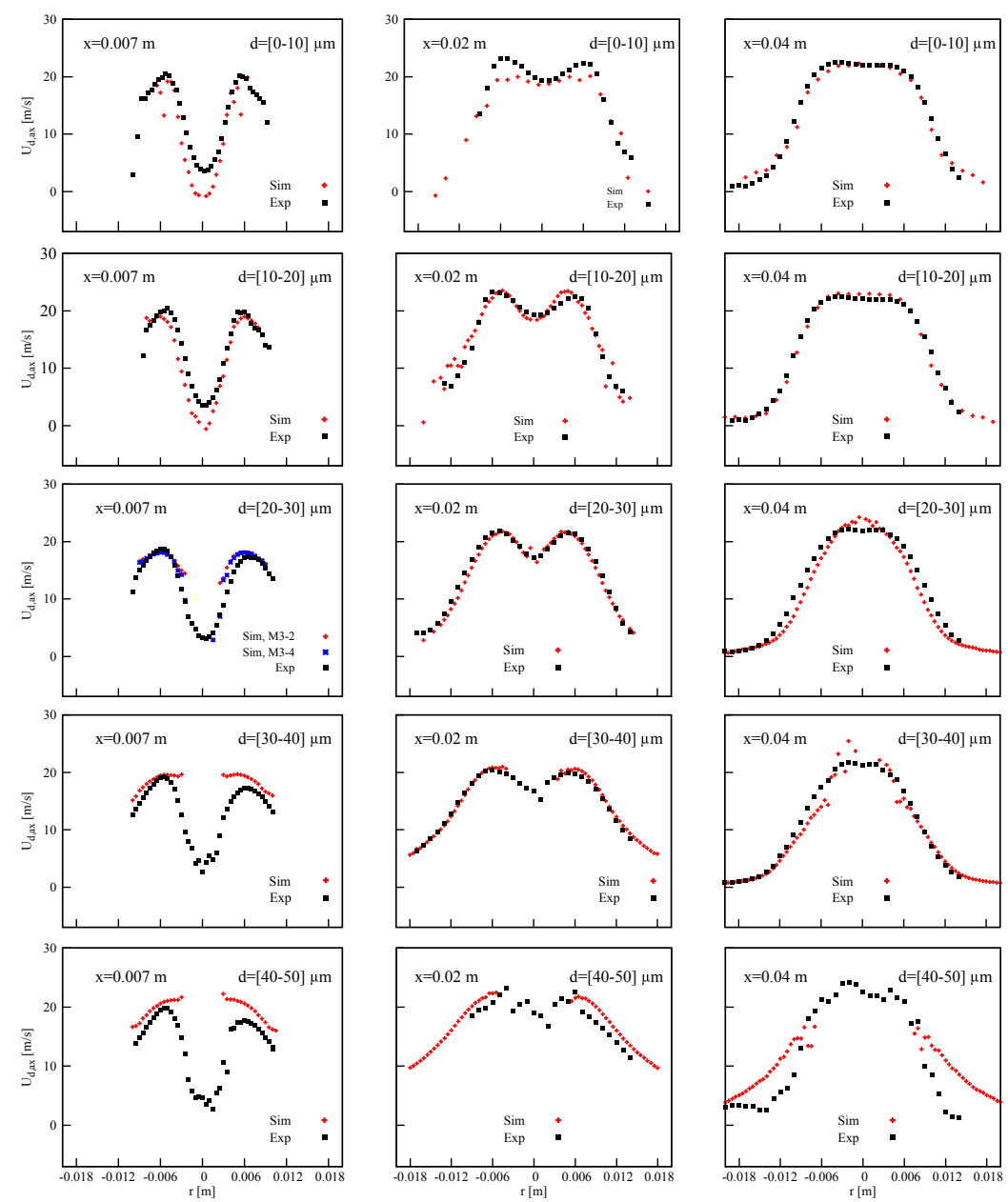

Fig. 16 Mean liquid axial velocity of droplet class sizes $d=[0-10 \mu m], d=[10-20 \mu m]$, $d=[20-30 \mu m], d=[30-40 \mu m]$ and $d=[40-50 \mu m]$ at $x=0.007 \mathrm{~m}, x=0.02 \mathrm{~m}, x=0.04 \mathrm{~m}$. Simulations results using M3 and $N p=2.2 \cdot 10^{6}$ parcels $/ s$ are in red crosses. Simulation results using M3 and $N p=4 \cdot 10^{6}$ parcels $/ \mathrm{s}$ are in blue crosses (only at $x=0.007 \mathrm{~m}$ ). Experiments are in black squares. Time averaging period is $0.95 \mathrm{~s}$.

$d_{p} \in[0-10 \mu m]$. The lower values observed in the simulations could explain the higher error observed for the mean liquid axial velocity at this location (Table 7 ). At $x=0.04 m$ the agreement is good for all the droplet size classes, except for the $d_{p} \in[40-50 \mu \mathrm{m}]$ where the predicted velocities are higher than the experimental values.

The droplet mean diameter, $d_{10}$, is reported in Figure 17 . The agreement between simulations and experiments is overall good. Differences using $\dot{N}_{p}=2.2$. $10^{6}$ parcels $/ \mathrm{s}$ and $\dot{N}_{p}=4 \cdot 10^{6}$ parcels $/ \mathrm{s}$ are negligible. The only deviations from the experimental data are seen at the center from $x=0.02 m$ onward. The lower values predicted in the simulations ( $6 \%$ error) are due to the aforementioned lack 
of statistics in the center for $d_{p}>30 \mu m$.
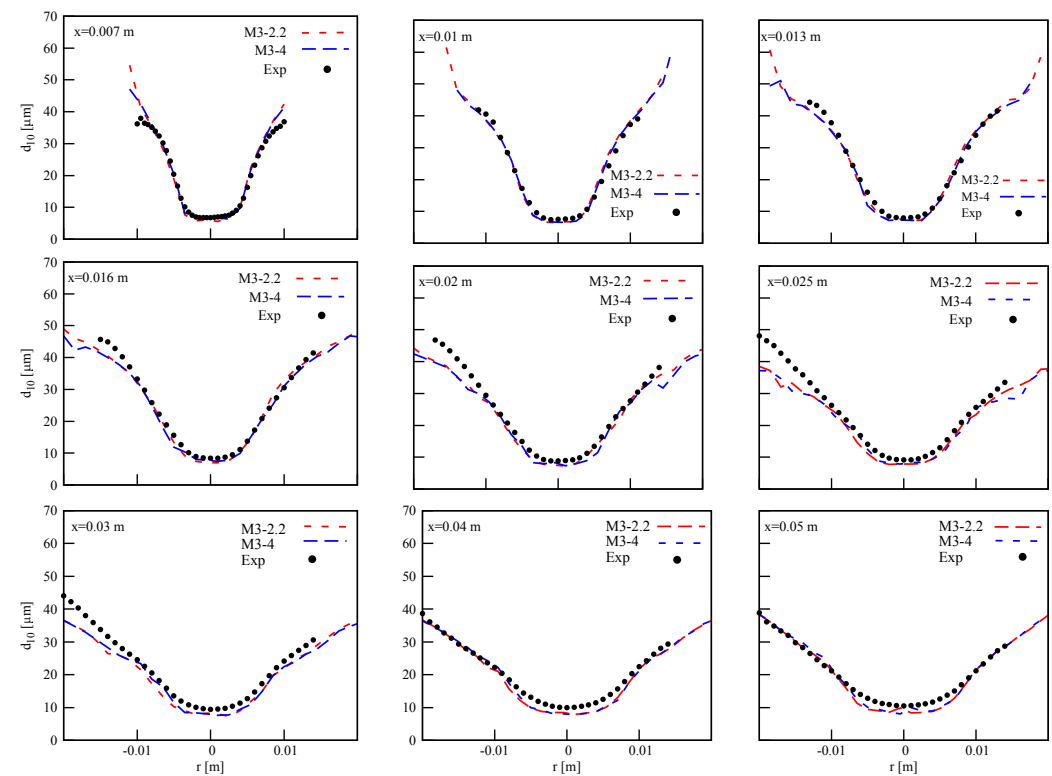

Fig. 17 Droplet mean diameter, $d_{10}$, radial distribution at different axial position (grid M3 with InjM model using $2.2 \cdot 10^{6}$ parcels/s and $4 \cdot 10^{6}$ ). Time averaging period is $0.95 \mathrm{~s}$. 
5.4 Injection Model

The results discussed so far were obtained with InjM. The methodology applied for the injection model InjM differs from the original ConeInjection model (InjOF) available in OpenFOAM-2.4.0. Particularly the spray angle, ranging from the imposed inner and outer values, is not selected randomly, as in the original OF implementaion (Sec 4.1). Rather, a normal distribution with mean value equal to 40 and variance equal to 8 is imposed.

In Figure 18, Favre mean averaged gas axial velocity, mean liquid axial velocity, mean droplet diameter and the histogram size based on number of parcels are reported at the axial locations $x \in[0.007 \mathrm{~m}, 0.02 \mathrm{~m}, 0.04 \mathrm{~m}]$. The modification of the injection model has no impact on the gaseous flow field. There is, however, impact on the liquid phase. Although at $x=0.007 \mathrm{~m}$ differences for the liquid phase are not significant, the parcels are distributed differently along the radial coordinate. With InjM more parcels are concentrated around $\theta=40^{\circ}$, corresponding to the defined mean value of the normal distribution and equal to the nominal spray angle in the experiments. Instead, with InjOF the parcels are uniformly distributed between the inner and outer angle. This leads to wider $d_{10}$ profiles. At $x=0.02 \mathrm{~m}$ the different parcels distribution along the radius has an impact on both the liquid mean axial velocity, $U_{d, a x}$ and the mean droplet diameter, $d_{10}$. Velocities with InjOF are lower at the outer branch, where the parcel distribution is more uniform than InjM model. Subsequently also the mean diameter is lower. At $x=0.04 m$ results are similar for both models for all the analyzed quantities. Here the two size histograms are almost identical. All the information imposed by the two injection methodologies is lost; parcels are driven by the gas phase and tend to be preferentially concentrated according to the gas phase motion. 

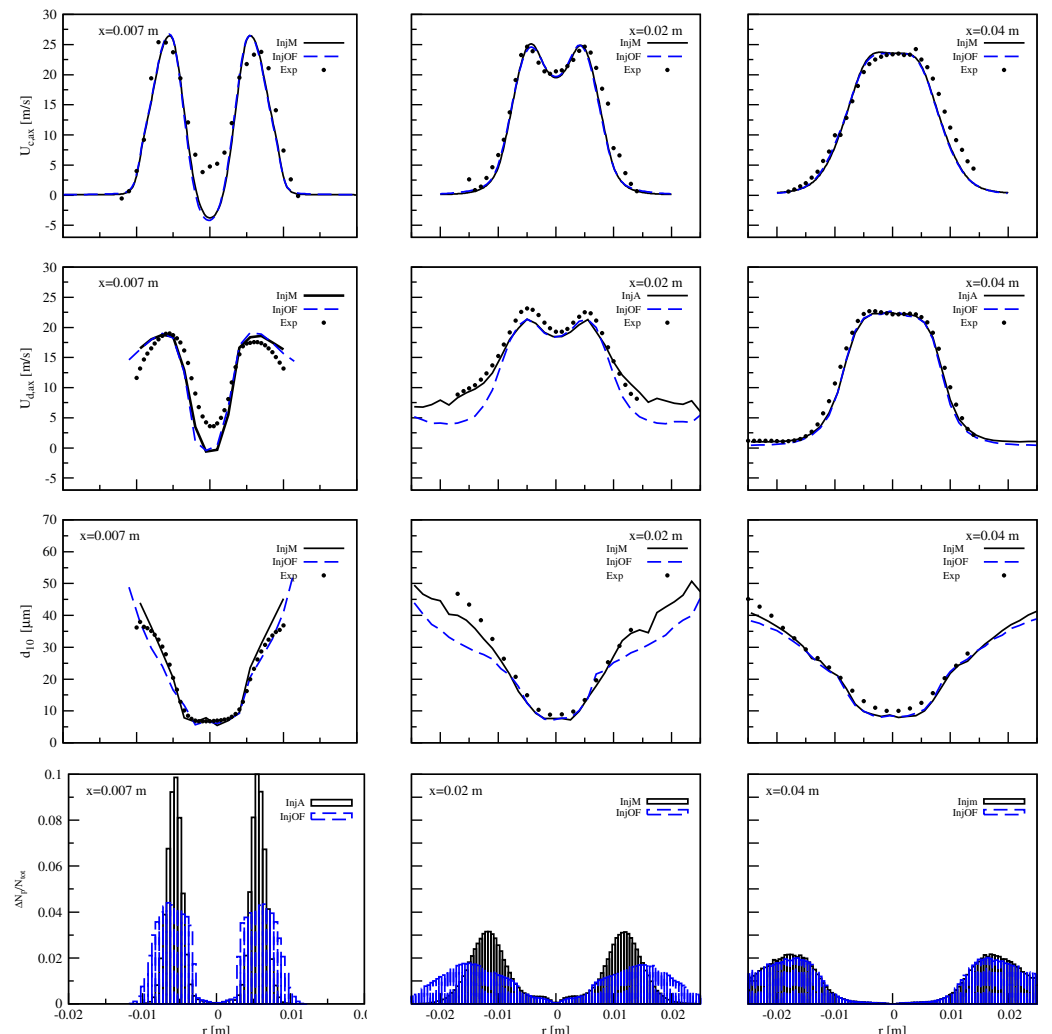

Fig. 18 Comparison between ConeInjection model (InjOF), modified ConeInjection model (InjM) and experimental data (Exp). From top to bottom: mean axial gas velocity $\left(U_{c, a x}\right)$, mean axial liquid velocity $\left(U_{d, a x}\right)$, droplet mean diameter $d_{10}$ and droplet size histogram based on number of droplets. InjM represented with black lines, InjOF in dashed blue lines and Experiments in black dots. Time averaging period is $0.95 \mathrm{~s}$. 


\section{Conclusions}

This study concerns Large Eddy Simulations (LES) of a turbulent spray jet in the Eulerian-Lagrangian 9EL) framework. The experimental case under study is part of the Coria Rouen Spray burner database, for which measurements of droplet fields and gas velocity are available both in reactive and non-reactive conditions. The latter are used in this work. An initial objective of the study was to identify, between four different grids, the cell size to be used for a reliable LES calculation when only the gas phase is simulated. The results have shown that for the case at hand grid convergence in terms of mean axial velocity is achieved if in the center a streamwise mesh size $\delta_{a x} \leq 0.2 \mathrm{~mm}$ and a spanwise mesh size $\delta_{\text {rad }} \leq 0.2 \mathrm{~mm}$ is used.

The assessment of reliable LES with only the gas phase allows another sensitivity study, for the liquid-gas phase simulations and based on the number of parcels to inject per second. In this study three different numbers are used: $\dot{N}_{p}=0.5 \cdot 10^{6}$ parcels $/ s, \dot{N}_{p}=2.2 \cdot 10^{6}$ parcels $/ s$ and $\dot{N}_{p}=4 \cdot 10^{6}$ parcels $/ \mathrm{s}$. The results for liquid-gas simulations on the same grids as for the only gas simulations are compared by looking at difference between spanwise mean axial velocities at different axial locations. The velocity offset showed the link between cell size and parcels number for $\dot{N}_{p}=0.5 \cdot 10^{6}$ parcels $/ \mathrm{s}$. Particularly by refining the mesh, the contribution from liquid to gas, in the center of the jet, becomes smaller. The offset values are unchanged refining the mesh for $\dot{N}_{p}=2.2 \cdot 10^{6}$ parcels $/ \mathrm{s}$ and $\dot{N}_{p}=4 \cdot 10^{6}$ parcels $/ \mathrm{s}$. Also scatter plots for Stokes number and relaxation times remain essentially unchanged, comparing $\dot{N}_{p}=2.2 \cdot 10^{6}$ parcels $/ \mathrm{s}$ and $\dot{N}_{p}=4 \cdot 10^{6}$ parcels $/ \mathrm{s}$. Based on the computational costs, $\dot{N}_{p}=2.2 \cdot 10^{6}$ parcels $/ \mathrm{s}$ is used for the comparison with the experimental data.

The droplets tendency to follow the gas phase motion has been analyzed with the particle Stokes number in three different spray jet regions: the near-nozzle region, the intermediate region and the far-downstream region. The most striking results are:

- Droplets having $d_{p} \leq 15 \mu \mathrm{m}$ always have $S t<1$, thus they follow the gas phase motion.

- The gaseous shear layer, from the intermediate region onward, behaves as separation front between droplets with $d_{p}<30 \mu \mathrm{m}$ and $d_{p}>30 \mu \mathrm{m}$.

Because the position of shear layer is sensitive to the grid size, this will vary according to the chosen LES mesh, influencing the liquid phase in substantial manner. This justifies the choice of the gas phase mesh first.

The comparison with experiments showed excellent agreement in the intermediate and far downstream region with errors on the maximum axial velocity as low as the $3 \%$ for the gas and $6 \%$ for the liquid phase.

In the near-nozzle region discrepancies between simulations and experiments are observed in the center. The simplification of the real injector geometry by an annular cylinder may be the cause of these deviations. In future research, the use of the real geometry will be adopted to examine whether results improve.

The mean droplet diameter is under-predicted in the center in the downstream region. This may be due to a strict separation at the gaseous shear layer between droplets with $d_{p}<30 \mu m$ and $d_{p}>30 \mu m$ or to difference in period duration to collect statistics between simulations and experiments. Further research should be done to investigate this. 
Finally the modified injection methodology showed a beneficial impact in the prediction of the experimental data for the liquid phase only, when compared with the original methodology as available in OpenFOAM.

A natural progression of this work is to analyze the reactive case. In this sense the study serves as a solid backbone for the study of the droplet evaporation, turbulence-chemistry interaction, auto-ignition and flame propagation, which introduces further modelling issues.

\section{Acknowledgements}

The Flemish Supercomputer Centre (VSC) (Flanders, Belgium) is acknowledged for the allocated computational time on the Tier-1 cluster Breniac installed in Leuven (Belgium) as well as the Tier-2 cluster Stevin installed in Ghent (Belgium).

\section{Funding}

This research has been funded by Ghent University (Belgium) through GOA project; [BOF16/GOA/004] (PREdiction of Turbulent REactive Flows http://www.pretref.ugent.be/)

\section{Compliance with Ethical Standards}

Conflict of Interest: The authors declare that they have no conflict of interest.

\section{References}

1. Beji, T., Ebrahimzadeh, S., Maragkos, G., Merci, B.: Numerical modelling of the interaction between water sprays and hot air jets - part ii: Two-phase flow simulations. Fire Safety Journal 96, 143-152 (2018). DOI 10.1016/j.firesaf.2018.01.006

2. Chigier, N.A., Beer, J.M.: The flow region near the nozzle in double concentric jets. Journal of Basic Engineering 86(4), 797-804 (1964). DOI 10.1115/1.3655957

3. Chigier, N.A., Beer, J.M.: Velocity and static-pressure distributions in swirling air jets issuing from annular and divergent nozzles. Journal of Basic Engineering 86(4), 788-796 (1964). DOI 10.1115/1.3655954

4. Crowe, C.T., Sommerfeld, M., Tsuji, Y.: Multiphase flows with droplets and particles. CRC Press, Boca Raton, Fla. (1998)

5. Elghobashi, S.: On predicting particle-laden turbulent flows. Applied Scientific Research 52(4), 309-329 (1994). DOI 10.1007/BF00936835

6. Garg, R., Narayanan, C., Lakehal, D., Subramaniam, S.: Accurate numerical estimation of interphase momentum transfer in lagrangianeulerian simulations of dispersed twophase flows. International Journal of Multiphase Flow 33, 1337-1364 (2007). DOI 10.1016/j.ijmultiphaseflow.2007.06.002

7. J. O'rourke, P., Subramaniam P. J. O'rourke, S., Subramaniam, S.: Numerical convergence of the kiva-3 code for sprays and its implications for modeling. Los Alamos Laboratory Report UR-98-5465 (1999)

8. Jenny, P., Roekaerts, D., Beishuizen, N.: Modeling of turbulent dilute spray combustion. Progress in Energy and Combustion Science 38(6), 846-887 (2012)

9. Kornev, N., Hassel, E.: Method of random spots for generation of synthetic inhomogeneous turbulent fields with prescribed autocorrelation functions. Communications in Numerical Methods in Engineering 23(1), 35-43 (2007). DOI 10.1002/cnm.880 
10. Lipowsky, J., Sommerfeld, M.: Time-dependent simulation of a swirling two phase flow using an anisotropic turbulent dispersion model. Proceedings of the ASME Fluids Engineering Division Summer Conference - 2005, Vol 1, Pts A and B pp. 677-686 (2005)

11. Lucchini, T., D'Errico, G., Ettorre, D.: Numerical investigation of the spray-meshturbulence interactions for high-pressure, evaporating sprays at engine conditions. International Journal of Heat and Fluid Flow 32(1), 285-297 (2011)

12. Ma, L., Roekaerts, D.: Modeling of spray jet flame under mild condition with non-adiabatic fgm and a new conditional droplet injection model. Combustion and Flame 165, 402-423 (2016)

13. Noh, D., Gallot-Lavallee, S., Jones, W.P., Navarro-Martinez, S.: Comparison of droplet evaporation models for a turbulent, non-swirling jet flame with a polydisperse droplet distribution. Combustion and Flame 194, 135-151 (2018). DOI 10.1016/j.combustflame.2018.04.018

14. Pesmazoglou, I., Kempf, A.M., Navarro-Martinez, S.: Large eddy simulation of particle aggregation in turbulent jets. Journal of Aerosol Science 111, 1-17 (2017). DOI 10.1016/j.jaerosci.2017.06.002

15. Pope, S.B.: Ten questions concerning the large-eddy simulation of turbulent flows. New Journal of Physics 6 (2004)

16. Rutland, C.J.: Large-eddy simulations for internal combustion engines - a review. International Journal of Engine Research 12(5), 421-451 (2011). DOI 10.1177/1468087411407248

17. Schiller, L., Naumann, A.: Über die grundlegende berechnung bei der schwerkraftaufbereitung. Ver. Dtsch. Inj 44, 318-320 (1933)

18. Schmidt, D.P., Bedford, F.: An analysis of the convergence of stochastic lagrangian/eulerian spray simulations. International Journal of Multiphase Flow 102, 95101 (2018)

19. Senecal, P.K., Pomraning, E., Richards, K.J., Som, S.: Grid-convergent spray models for internal combustion engine computational fluid dynamics simulations. Journal of Energy Resources Technology-Transactions of the Asme 136(1) (2014)

20. Senecal, P.K., Pomraning, E., Xue, Q., Som, S., Banerjee, S., Hu, B., Liu, K., Deur, J.M.: Large eddy simulation of vaporizing sprays considering multi-injection averaging and grid-convergent mesh resolution. Journal of Engineering for Gas Turbines and PowerTransactions of the Asme 136(11) (2014)

21. Shum-Kivan, F., Santiago, J.M., Verdier, A., Riber, E., Renou, B., Cabot, G., Cuenot, B.: Experimental and numerical analysis of a turbulent spray flame structure. Proceedings of the Combustion Institute 36(2), 2567-2575 (2017)

22. Ukai, S.: Conditional moment closure modelling of turbulent spray flames. Ph.D. thesis, University of Stuttgart (2014)

23. Verdier, A.: Experimental study of dilute spray combustion. Ph.D. thesis, University of Normandy, CORIA laboratory (2017)

24. Verdier, A., Santiago, J.M., Vandel, A., Godard, G., Cabot, G., Renou, B.: Local extinction mechanisms analysis of spray jet flame using high speed diagnostics. Combustion and Flame 193, 440-452 (2018). DOI 10.1016/j.combustflame.2018.03.032

25. Verdier, A., Santiago, J.M., Vandel, A., Saengkaew, S., Cabot, G., Grehan, G., Renou, B.: Experimental study of local flame structures and fuel droplet properties of a spray jet flame. Proceedings of the Combustion Institute 36(2), 2595-2602 (2017)

26. Weller, H.G., Tabor, G., Jasak, H., Fureby, C.: A tensorial approach to computational continuum mechanics using object-oriented techniques. Computers in Physics 12(6), 620631 (1998). DOI Doi 10.1063/1.168744

27. Yoshimoto, H., Goto, S.: Self-similar clustering of inertial particles in homogeneous turbulence. Journal of Fluid Mechanics 577, 275-286 (2007). DOI 10.1017/S0022112007004946 